\title{
Lidil
}

Revue de linguistique et de didactique des langues

$62 \mid 2020$

Recherches actuelles en didactique du lexique: avancées, réflexions, méthodes

\section{Des mots pour dire et écrire une ruse : influences de pratiques orales sur les productions écrites d'élèves de 10 à 13 ans}

Words to Tell and Write a Trick: Influences of Oral Activities on the Written

Work of Pupils Aged from 10 to 13

\section{Christine Vénérin-Guénez}

\section{OpenEdition}

Journals

Édition électronique

URL : http://journals.openedition.org/lidil/8323

DOI : $10.4000 /$ lidil.8323

ISSN : 1960-6052

Éditeur

UGA Éditions/Université Grenoble Alpes

Édition imprimée

ISBN : 978-2-37747-226-0

ISSN : $1146-6480$

Référence électronique

Christine Vénérin-Guénez, «Des mots pour dire et écrire une ruse : influences de pratiques orales sur les productions écrites d'élèves de 10 à 13 ans », Lidil [En ligne], 62 | 2020, mis en ligne le 03 novembre 2020, consulté le 10 décembre 2020. URL : http://journals.openedition.org/lidil/8323 ; DOI : https:// doi.org/10.4000/lidil.8323

Ce document a été généré automatiquement le 10 décembre 2020.

(C) Lidil 


\title{
Des mots pour dire et écrire une ruse : influences de pratiques orales sur les productions écrites d'élèves de 10 à 13 ans
}

\author{
Words to Tell and Write a Trick: Influences of Oral Activities on the Written \\ Work of Pupils Aged from 10 to 13
}

Christine Vénérin-Guénez

\section{Introduction}

1 Notre projet de recherche s'inscrit dans la continuité de nos travaux en didactique de l'écriture, sous l'angle des choix lexicaux, dans le prolongement d'une recherche entreprise de 2011 à 2015 au sein du laboratoire STIH (Paris Sorbonne). Le projet « Des pratiques de l'oral au service de l'écriture » a fait l'objet d'une convention de recherche entre l'Académie de La Réunion et l'Université de La Réunion. Des angles d'analyse sont envisagés dans une double perspective, didactique disciplinaire et didactique professionnelle.

2 Notre recherche empirique vise à mesurer l'influence de plusieurs séances lexicales orales sur le réinvestissement en production écrite. Le dispositif favorise la conscience réflexive sur les choix lexicaux, en réception et en production, donc le développement des compétences lexicale et métalexicale. Nous rendons compte d'un dispositif expérimental mis en œuvre dans six classes réunionnaises, sur trois niveaux (CM2, $6^{\mathrm{e}}$, $5^{\mathrm{e}}$ ), entre février et mai 2018. L'ensemble du dispositif a concerné un peu plus de 150 élèves. Le dispositif repose sur les mêmes contes entendus et des rappels de récit écrits, donc un substrat narratif commun qui facile les interactions orales en classe et les comparaisons de faits de langue dans les productions écrites. Le recueil de données analysées comporte 463 productions écrites et huit heures de vidéos enregistrées dans 
quatre classes. De multiples angles d'analyse sont envisagés dans une double perspective, didactique disciplinaire par l'entrée lexicale d'une part, didactique professionnelle d'autre part, volet qui sera esquissé dans cette contribution et développé dans d'autres travaux.

3 Après avoir présenté notre dispositif, nous décrirons tour à tour ses effets sur les apprentissages lexicaux puis ses bénéfices plus généraux pour les élèves et les enseignantes.

\section{Le cadre théorique}

4 Nous nous plaçons dans le cadre des travaux initiés par Hayes et Flower (1980) sur les processus de production écrite qui ont engendré une abondante littérature autour des trois grands axes de leur modèle (planification, mise en texte, révision), des origines des difficultés rencontrées par les scripteurs au cours de la rédaction d'un texte, des conditions d'amélioration des productions. Nous avons étudié les stratégies dont usent les scripteurs pour contourner les difficultés imposées par les limites de leurs capacités mnésiques et/ou de leurs ressources langagières (Vénérin-Guénez, 2012, 2017, 2018a) dans un dispositif expérimental de doubles rappels du même récit à une semaine d'intervalle. Nous avons montré l'intérêt didactique et pédagogique d'entretiens d'explicitations lexicales menés dans les classes à partir de choix lexicaux repérés par le chercheur dans les productions des élèves (Vénérin-Guénez, 2018b ; Vénérin-Guénez \& Gaillat, 2018). Les objectifs de ces entretiens sont multiples : comprendre les choix lexicaux des élèves pour les aider à les améliorer, sensibiliser les élèves à la structure syntaxico-sémantique des mots et donc développer leur conscience réflexive pour améliorer la révision de leurs productions écrites.

5 Notre recherche à orientation didactique articule les apprentissages lexicaux à la production verbale et s'appuie sur des travaux dédiés à l'intégration de la didactique du lexique dans celle de l'écriture (Grossmann \& Plane, 2008) et sur ceux menés à l'INRP sur le rôle des interactions orales dans la construction des apprentissages (GarciaDebanc \& Plane, 2004; Grandaty \& Turco, 2001). Une réflexion de Grossmann et Boch (2003) nous a amenée à construire un dispositif articulant lecture, écriture et oral. Dans l'introduction de leur article, les auteurs exposent les raisons qui les motivent à "articuler une didactique de l'écriture et une didactique du lexique» au travers du lexique de l'émotion et des sentiments. Les auteurs s'appuient sur l'idée qu'«un entrainement prenant en compte le lexique peut avoir des répercussions positives, si cet entrainement est effectué dans une perspective de productions de textes " et constatent qu'« on a peu étudié jusqu'à présent la place de la compétence lexicale et métalexicale dans le domaine de la production de texte, ainsi que l'impact que pourrait avoir un entrainement spécifiquement centré sur le développement des compétences métalexicales » (p. 117-118).

6 Ces considérations nous ont amenée à construire un dispositif expérimental fondé sur un entrainement régulier à partir de plusieurs rappels de récit et d'entretiens métalexicaux, en vue d'un travail d'écriture d'invention. Il inclut des phases orales centrées sur le développement de compétences lexicales et métalexicales. L'oral, rappelons-le, est un moyen d'apprentissage efficace dont les fonctions didactiques sont formulées en ces termes par Garcia-Debanc et Plane (2004) : "La verbalisation et les interactions obligent à restructurer les champs conceptuels, à intégrer des 
apports d'informations, à intégrer dans son propre discours des éléments du discours d'autrui. »(p. 33) Au cœur du dispositif s'articulent plusieurs domaines de l'enseignement-apprentissage $d u$ français: le langage oral, la lecture en réception orale, l'écriture d'un rappel de récit, qui sont autant de médiations au service du développement des compétences lexicale et métalexicale. En d'autres termes, notre dispositif relève de l'« apprentissage intégré » de l'oral, de la lecture et de l'écriture, formule que nous empruntons à Grossmann (2018).

\section{Le contexte de passation}

7 Les six classes impliquées dans l'expérimentation sont situées dans la ville de SaintDenis de La Réunion, dans des écoles et collèges différents; toutes sont des classes à dominante créolophone. Quatre classes participent au dispositif complet, lectures entendues, rappels de récit écrits, séances lexicales orales filmées et écriture d'invention : un $\mathrm{CM} 2$, deux $6^{\mathrm{e}}$, une $5^{\mathrm{e}}$ (92 élèves). Deux classes témoins, un CM2 et une $6^{\mathrm{e}}$ (42 élèves), ne bénéficient pas de séances lexicales orales. Sur les 152 élèves des six classes, 134 élèves ( 66 filles et 68 garçons) ont été retenus. Les autres ont été exclus des analyses soit parce qu'ils sont absents à au moins deux écrits, et/ou à au moins deux séances lexicales orales, soit parce qu'ils n'ont pas voulu écrire ou n'ont pas réussi à écrire.

8 Les enseignantes, reconnues et impliquées dans différentes actions de formation académique, ont été sollicitées par leur inspecteur. Elles ont plusieurs années d'expériences d'enseignement: Danielle (CM2-D), 13 années; Clarice (CM2-C classe témoin), 17 années ; Cécile (6e-C (1) et (2)), 18 années ; Anne-Laure (5e), 16 années. Marie $\left(6^{\mathrm{e}}-\mathrm{M}\right)$ est la moins expérimentée, titulaire depuis 2 ans.

9 Nous avons choisi de faire participer des classes dont les élèves sont à dominante créolophone. En effet, même si le champ investigué est bien celui de la didactique du lexique et de l'écriture en français langue première, l'enjeu de recherche est enrichi par la problématique de l'enseignement-apprentissage en contexte plurilingue réunionnais. Nous nous sommes attachée, dans une autre contribution (Vénérin-Guénez, 2020), à observer et analyser l'influence du contexte sociolinguistique réunionnais dans les productions écrites et les séances orales.

10 Quelques aléas ont perturbé les programmations établies dans chaque classe à partir de la mi-février 2018. Une tempête tropicale a contraint à la fermeture des établissements scolaires début mars. Le rythme de passation sur trois semaines consécutives n'a pas pu être respecté dans la $6^{\mathrm{e}}-\mathrm{M}$. Par ailleurs, les séances orales filmées étant programmées le vendredi, l'intégralité des élèves de deux classes, la $6^{\mathrm{e}}-\mathrm{C}$ et la $5^{\mathrm{e}}$, n'a pas pu participer à la première séance orale. Plus de la moitié des élèves étaient absents le vendredi de Pâques pour participer aux cérémonies religieuses. Enfin, dix élèves de $6^{\mathrm{e}}-\mathrm{M}$ n'ont pas $\mathrm{pu}$ assister à l'audition du troisième conte et son rappel écrit en raison de leur engagement dans un concours académique. Les perturbations engendrées par le contexte climatique et socio-culturel réunionnais atténuent sans doute les effets supposés bénéfiques d'un entrainement régulier autour d'activités lexicales.

11 Quant aux séances lexicales orales (désormais SLO), le protocole prévoit de sélectionner des unités lexicales (désormais UL) concernant la ruse et les sentiments. Mais dans la $6^{\mathrm{e}}-\mathrm{M}$, l'expression des sentiments et des émotions n'a été abordée dans aucune des trois SLO, seulement dans la première SLO dans la $6^{\mathrm{e}}-\mathrm{C}(1)$, pas du tout dans la première SLO 
du CM2-D. Compte tenu de la plus faible durée globale d'activités sur le vocabulaire des sentiments, et potentiellement du plus faible impact en réinvestissement dans l'écriture d'invention, dans le cadre de cette contribution, nous avons opté pour une analyse comparative des performances lexicales centrées sur le vocabulaire de la ruse.

\section{La méthodologie}

\subsection{Les contes entendus}

12 Le dispositif expérimental comporte trois contes sur le thème de la ruse, deux contes africains: "Le lièvre astucieux », "Les trois ruses de la gazelle ${ }^{1}$ " et un conte de Perrault "Le chat botté ». Le fait que le conte animalier soit un genre familier aux élèves et que la structure narrative de réitération de la ruse sont des facteurs de facilitation mémorielle pour aider les élèves à construire une représentation mentale de l'univers de référence. Les trois récits ont été légèrement adaptés à partir de leur version originale par quelques suppressions pour raccourcir le texte et pour insérer davantage d'éléments lexicaux dénotant la ruse et les affects de la joie et de la colère. La thématique de la ruse et le genre du conte s'insèrent dans les programmes de français de 2018 pour les trois niveaux. Le dispositif perturbe peu la programmation des enseignements prévue dans les textes institutionnels.

\subsection{Le rappel de récit}

Des travaux en psycholinguistique ont éclairé les contraintes inhérentes à la réception d'un texte et à la production écrite ou orale d'un rappel de récit, en particulier ceux de Le Ny (1989) et de Denhière et Baudet (1992) qui reprennent et développent la théorie de la compréhension de texte à partir de modèles mentaux définis initialement par Johnson-Laird (1983) et Van Dijk et Kinstch (1983). L'activité cognitive du lecteur ou de l'auditeur est orientée vers la représentation du contenu, activité de compréhension complexe qui active des schèmes mentaux préexistants en mémoire, conjuguée au stockage d'informations.

Dans le domaine de la compréhension en lecture, Goigoux et Cèbe (2018) exposent les grands principes théoriques qui guident leur dispositif didactique Lectorino \& Lectorinette en insistant sur le développement des compétences narratives en production, de la compétence lexicale et en mettant au premier plan la compréhension des états mentaux des personnages qui permettent le développement des compétences inférentielles. Les auteurs signalent que «la compréhension de l'implicite repose sur celle de l'identité psychologique et sociale des personnages, de leurs mobiles, de leurs systèmes de valeur, de leurs affects, de leurs connaissances. Elle exige que les élèves identifient et sachent nommer leurs sentiments, leur caractère, leurs croyances, mais aussi leurs buts, car ce sont ces derniers qui motivent leurs actions. » (p. 19)

Le rappel de récit est une activité préconisée dans le dispositif de Goigoux et Cèbe (2018). On demande à un élève de lire ou d'écouter une histoire et de la redire avec ses propres mots (Giasson, 1996). Cette activité langagière est utilisée comme stratégie d'enseignement pour évaluer la compréhension en lecture, mais aussi comme outil pour travailler la compréhension du langage écrit et développer le langage oral, particulièrement en maternelle (Brigaudiot, 2000). Même si des segments de l'histoire 
sont parfois mémorisés, le rappel de récit, écrit ou oral, s'accompagne le plus souvent de reformulations, de restructurations des informations, de sélections et d'ajouts, autant de paramètres observables et comparables dans le corpus que nous avons recueilli.

Dans notre dispositif, le substrat initial commun des trois contes entendus facilite les interactions verbales dans la classe lors des séances lexicales orales puisque les élèves connaissent les mêmes univers narratifs. Nous avons rappelé supra l'importance des états mentaux, de la dénomination des sentiments et des personnages. Le récit de ruse implique nécessairement un trompeur et un trompé, et donc implicitement les sentiments afférents à la situation psychologique des personnages. Le lexique des émotions et des sentiments est également le prisme adopté par Grossmann et Boch (2003, p. 121) parce que l'écriture d'un récit fictionnel suppose que le scripteur soit en mesure de traduire un point de vue, ce qui passe généralement par la traduction des sentiments des personnages, et parce que ce lexique abstrait est assez peu mobilisé dans sa diversité dans les productions d'élèves.

\subsection{Le protocole de passation}

17 Le dispositif complet comporte sept phases comportant trois types d'activités décrites un peu plus loin : trois phases de lecture entendue suivie immédiatement d'un rappel de récit écrit, une par semaine pendant trois semaines consécutives; trois phases filmées de séances lexicales orales deux à trois jours après chaque rappel écrit (sauf pour les classes témoins), une phase d'écriture d'invention d'une ruse deux semaines plus tard environ. L'ordre de découverte des contes est le suivant: "Le lièvre astucieux » (862 mots), "Le chat botté » (1384 mots), "Les trois ruses de la gazelle » (1022 mots) ${ }^{2}$. Le sujet d'écriture d'invention a porté sur la quatrième ruse de la gazelle.

Pour la mise en œuvre des trois rappels écrits, l'enseignante lit le conte en début de semaine, les élèves n'ont pas le texte sous les yeux. Ils sont avertis qu'ils n'entendront le texte qu'une seule fois, sans prise de note et qu'ils doivent se concentrer pour bien écouter le conte, le comprendre et le mémoriser. Ils savent qu'ils devront écrire ce dont ils se souviennent le plus précisément possible, juste après l'audition, pour une durée souple, entre 30 et 45 minutes ${ }^{3}$. L'enseignante leur précise que leur travail n'est pas évalué et contribue à des données de recherche, que les ratures et les erreurs orthographiques ne sont pas prises en compte dans l'expérience, que la longueur est libre. Ces différents paramètres annoncés visent à motiver les élèves, à favoriser la participation et le plaisir d'écrire sans crainte de sanction d'aucune sorte. Grâce à ces conditions, le rappel de récit affranchit l'élève partiellement des contraintes d'invention et de planification.

Pour la préparation des séances lexicales orales de la fin de chacune des trois semaines consécutives, l'enseignante repère dans les productions écrites les choix lexicaux qui seront les objets de discussion collective. Les critères de repérage lui sont explicités préalablement pour la guider : relever des mots ou expressions appartenant au champ lexical de la ruse (verbes, noms, adjectifs) et/ou dénotant des sentiments et émotions (joie, colère, peur, etc.). Il s'agit de préparer des questions variées portant sur des réussites, des maladresses ou des erreurs lexicales. Certaines questions seront ciblées et adressées à un ou plusieurs élèves : il faut donc noter le nom des " auteurs » des choix lexicaux. D'autres questions plus génériques peuvent être ouvertes à tous, telles que 
celles portant sur la collecte de synonymes, de mots de la même famille, sur le niveau de langue, etc. L'enseignante invite chacun à participer, même s'il n'avait pas fini d'écrire, ou pas tout mémorisé, ou pas tout compris. Les questions génériques font appel à des connaissances hors texte et permettent donc la participation des élèves absents à la lecture du conte. Consigne est donnée aux enseignantes de nommer un ou plusieurs élèves, mais de laisser aussi intervenir ceux qui voudraient compléter une explication, donner leur avis, proposer un autre choix lexical. Les séances orales peuvent durer 30 à 45 minutes et sont filmées.

La dernière phase écrite se déroule au retour des vacances scolaires de mars. L'enseignante annonce aux élèves qu'ils vont devoir écrire eux-mêmes une ruse, juste après avoir réécouté le conte "Les trois ruses de la gazelle ». Lors de la passation, elle donne la consigne suivante : «Inventez une quatrième ruse de la gazelle qui promet à la fin du conte de se venger du léopard. Expliquez comment elle s'y prend pour tromper encore une fois le léopard. " Il est convenu que l'enseignante ne demande pas à ce que les élèves utilisent les unités lexicales (UL) travaillées en classe puisque l'un des objectifs est de mesurer l'influence des séances orales sur le réemploi spontané.

Dans cette perspective de mesure, les élèves des deux classes témoins (CM2-C et $6^{\mathrm{e}}$ $C(2))$ ne bénéficient pas de séances lexicales orales. Le CM2-C a entendu les trois contes et réalisé trois rappels de récit, la $6^{\mathrm{e}}-\mathrm{C}$ (2) n'a entendu que le troisième conte et réalisé le rappel du récit. Tous ont produit un écrit d'invention d'une quatrième ruse de la gazelle.

\section{Les effets du dispositif didactique sur les apprentissages lexicaux}

\subsection{L'analyse quantitative de la longueur des productions écrites}

Les premières analyses quantitatives montrent des disparités de performances entre les classes. Les productions écrites des élèves du CM2-C témoin, dans les trois rappels, sont toujours plus longues que celles des quatre autres classes. Cet indicateur quantitatif nous a intriguée. Un bref entretien avec l'enseignante nous a permis de comprendre que le choix de la classe comme terrain d'investigation n'avait pas été judicieux. L'enseignante suivait les élèves depuis trois ans, et insistait particulièrement, entre autres, sur l'apprentissage de l'écriture. Les compétences scripturales des élèves de cette classe sont plus élevées que dans les classes ordinaires, vraisemblablement grâce à un enseignement-apprentissage régulier, tant pour l'appropriation du schéma narratif que pour la rapidité du geste grapho-moteur. Le tableau 1 ci-dessous montre les écarts de longueur moyenne en mots des différentes classes.

Tableau 1. - Analyse comparative des longueurs moyennes des productions écrites (en nombre de mots).

\begin{tabular}{|l|l|l|l|l|l|}
\hline 134 & $\begin{array}{l}\text { «Le lièvre } \\
\text { élèves retenus }\end{array}$ & $\begin{array}{l}\text { «Le chat } \\
\text { botté » }\end{array}$ & $\begin{array}{l}\text { «Les trois ruses de } \\
\text { la gazelle » }\end{array}$ & $\begin{array}{l}\text { Ruse } \\
\text { inventée }\end{array}$ & $\begin{array}{l}463 \\
\text { productions }\end{array}$ \\
\hline CM2-D & 225 & 180 & 206 & 129 & 78 \\
\hline
\end{tabular}




\begin{tabular}{|l|l|l|l|l|l|}
\hline $6^{\mathrm{e}-C}(1)$ & 221 & 196 & 225 & 178 & 95 \\
\hline $6^{\mathrm{e}-\mathrm{M}}$ & 238 & 207 & 246 & 177 & 81 \\
\hline $5^{\mathrm{e}}$ & 278 & 240 & 216 & 205 & 82 \\
\hline $\mathrm{CM} 2-\mathrm{C}$ témoin & 327 & 351 & 312 & 185 & 95 \\
\hline $\begin{array}{l}6^{\mathrm{e}}- \\
\mathrm{C}(2) \text { témoin }\end{array}$ & - & - & 166 & 123 & 32 \\
\hline
\end{tabular}

On constate que les élèves du CM2-C témoin sont des scripteurs plus performants, sur le plan de la longueur, dès le premier rappel de récit: 327 mots versus 225, 221, 238, 278 mots pour les autres classes, même la $5^{\mathrm{e}}$. On constate également que la $6^{\mathrm{e}}-\mathrm{C}$ (2) témoin, non entrainée par les deux premiers rappels, présente une faible performance de longueur : 166 mots versus 206, 225, 246, 216, 312 mots.

La longueur des productions écrites peut être sans doute corrélée à un entrainement régulier à l'écriture, à l'âge des élèves puisque le dispositif est expérimenté sur trois niveaux de classe, et à la capacité de concentration du public scolaire. Mais les facteurs de longueur du conte entendu, de difficultés lexicales ou narratives, sa drôlerie, la malice du protagoniste, etc., entrent aussi en ligne de compte. Nous avons interrogé les élèves sur le conte qui leur avait le plus plu ou au contraire le moins plu, et pourquoi. Voici un aperçu des éléments de réponses fournis :

- CM2-D : le conte 2 « connu » est jugé « long et compliqué »;

- $6^{\mathrm{e}}-\mathrm{C}$ : le conte 2 «connu » est le moins apprécié car «trop long», le conte 3 est le plus apprécié ;

- $6^{\mathrm{e}}-\mathrm{M}$ : le conte 1 a «enthousiasmé » les élèves, le conte 2 «connu » est jugé «long et difficile $»$;

- $5^{\mathrm{e}}$ : le conte 2 «connu» a rendu les élèves "plus confiants", le conte 3 est «le plus amusant »;

- CM2-C témoin : le conte 1 est le plus apprécié ; le conte 2 « connu » est le moins apprécié.

Le conte 2 « Le chat botté » n'emporte pas les faveurs. Il est vrai qu'il comporte plus de phases narratives, un préambule assez long avant d'arriver aux ruses du chat botté. Le fait d'être connu ne rend pas l'activité de rappel plus facile, sauf pour la $5^{\mathrm{e}}$ et le $\mathrm{CM} 2-$ C témoin. Dans trois des classes, on constate une baisse de performance quantitative par rapport aux deux autres rappels : 180, 196, 207 mots.

Les analyses comparatives doivent être relativisées compte tenu de plusieurs facteurs circonstanciels (voir supra), de la longueur inégale des productions écrites et de la qualité narrative des rappels. En effet, plus un rappel est précis et complet, plus le nombre d'UL réemployées est susceptible d'être élevé. Ainsi, le conte " Le chat botté » a été moins apprécié, les rappels de ce récit ont été globalement plus courts et souvent très incomplets. Cela pourrait expliquer par exemple le faible taux de réemplois d'UL dénotant la ruse. 


\subsection{L'analyse quantitative des performances lexicales dans les productions écrites}

Nous observerons tour à tour le nombre d'UL dénotant la ruse, employées dans les écrits d'invention d'une ruse (dernière phase du dispositif expérimental), puis le nombre d'UL dans les rappels de récits successifs de la première phase. L'hypothèse était qu'un entrainement régulier spécifiquement centré sur le développement de la compétence lexicale à l'écrit et à l'oral peut avoir des effets bénéfiques sur le réinvestissement en production écrite spontanée.

Les premières analyses des écrits d'invention de ruse ne montrent pas globalement une nette efficacité du travail lexical oral de la thématique pendant trois SLO en classe. Le CM2-C témoin sans SLO emploie sensiblement autant de mots de la ruse que les quatre classes avec SLO : $69 \%$ versus $62 \%$. Sans doute faut-il y voir l'impact des lectures successives de contes et faut-il tenir compte des aléas de passation des SLO mentionnés supra.

Néanmoins, les résultats n'invalident pas l'expérimentation. En revanche, si nous affinons la comparaison en proposant les pourcentages (arrondis), classe par classe, dans le tableau 2, deux classes ont des résultats similaires (65\%). Les faibles performances du CM2-D en particulier font baisser la moyenne. Les élèves de cette classe sont, dès le départ, moins performants dans la longueur des rappels de récits que toutes les autres classes, et ce, malgré leur implication très sérieuse.

Tableau 2. - Comparaison par classe du réinvestissement du vocabulaire de la ruse en écrit d'invention.

\begin{tabular}{|l|l|l|l|}
\hline & $\begin{array}{l}\text { Nombre d'écrits } \\
\text { d'invention comportant } \\
\text { aumoins une UL dénotant } \\
\text { la ruse }\end{array}$ & $\begin{array}{l}\text { Nombre d'écrits } \\
\text { d'invention } \\
\text { produits par } \\
\text { classe }\end{array}$ & $\begin{array}{l}\text { Pourcentage } \\
\text { d'invention employant } \\
\text { au moins une UL dénotant } \\
\text { la ruse }\end{array}$ \\
\hline CM2-D & 11 & 20 & $55 \%$ \\
\hline $6^{e}-C$ & 15 & 23 & $65 \%$ \\
\hline $6^{e}-M$ & 15 & 23 & $65 \%$ \\
\hline $5^{\text {e }}$ & 11 & 18 & $61 \%$ \\
\hline Témoin sans SLO & 18 & 26 & $69 \%$ \\
\hline $\begin{array}{l}\text { Témoin sans SLO, } \\
1 \text { seul R }\end{array}$ & 11 & 13 & $69 \%$ \\
\hline
\end{tabular}

En revanche, les SLO semblent avoir une efficacité sur l'emploi d'UL dénotant la ruse dans les rappels de récits 2 (désormais $\mathrm{R} 2$ ) et 3 (désormais $\mathrm{R} 3$ ), comme le montre le tableau 3. Le CM2-C témoin présente des performances moins bonnes. Nous pouvons supposer que les SLO dédiées au vocabulaire de la ruse influencent de façon bénéfique les choix lexicaux de semaine en semaine. 
Tableau 3. - Comparaison par classe d'emplois du vocabulaire de la ruse dans les trois rappels.

\begin{tabular}{|c|c|c|c|c|c|c|c|c|c|}
\hline \multirow{2}{*}{$\begin{array}{l}\text { N.B. En R1, pas de } \\
\text { SLO préalable } \\
\text { CM2-D }\end{array}$} & \multicolumn{3}{|c|}{$\begin{array}{l}\text { Nombre de rappels } \\
\text { comportant au moins } \\
\text { une UL / } \\
\text { nombre de R1 / } \\
\text { pourcentage (arrondi) }\end{array}$} & \multicolumn{3}{|c|}{$\begin{array}{l}\text { Nombre de rappels } \\
\text { comportant au moins } \\
\text { une UL / } \\
\text { nombre de R2 / } \\
\text { pourcentage (arrondi) }\end{array}$} & \multicolumn{3}{|c|}{$\begin{array}{l}\text { Nombre de rappels } \\
\text { comportant au moins } \\
\text { une UL / } \\
\text { nombre de R3 / } \\
\text { pourcentage (arrondi) }\end{array}$} \\
\hline & 9 & $\begin{array}{l}20 \\
\text { R1 }\end{array}$ & $45 \%$ & 7 & $20 \mathrm{R} 2$ & $35 \%$ & 10 & $18 \mathrm{R} 3$ & $56 \%$ \\
\hline $6^{\mathrm{e}}-\mathrm{C}(1)$ & 14 & $\begin{array}{l}24 \\
\text { R1 }\end{array}$ & $58 \%$ & 6 & $23 \mathrm{R} 2$ & $26 \%$ & 13 & $25 \mathrm{R} 3$ & $52 \%$ \\
\hline $6^{e}-M$ & 15 & $\begin{array}{l}23 \\
\text { R1 }\end{array}$ & $65 \%$ & 8 & $22 \mathrm{R} 2$ & $36 \%$ & 7 & $13 \mathrm{R} 3$ & $54 \%$ \\
\hline $5^{\mathrm{e}}$ & 15 & $\begin{array}{l}22 \\
\text { R1 }\end{array}$ & $68 \%$ & 19 & $23 \mathrm{R} 2$ & $39 \%$ & 13 & $19 \mathrm{R} 3$ & $68 \%$ \\
\hline Témoin sans SLO & 16 & $\begin{array}{l}23 \\
\text { R1 }\end{array}$ & $70 \%$ & 8 & $24 \mathrm{R} 2$ & $33 \%$ & 11 & $22 \mathrm{R} 3$ & $50 \%$ \\
\hline $\begin{array}{l}\text { Témoin sans SLO, } \\
1 \text { seul R }\end{array}$ & - & & & - & & & 3 & $16 \mathrm{R} 3$ & $19 \%$ \\
\hline
\end{tabular}

Nous constatons que le $\mathrm{CM} 2-\mathrm{C}$ témoin est plus performant au premier rappel (désormais R1) (70\%), en particulier par rapport aux CM2-D (45\%) et $6^{\mathrm{e}-\mathrm{C}}(1)$ (58 \%). Cependant cette performance élevée disparait en $\mathrm{R} 2^{4}$ et $\mathrm{R} 3$. Nous pouvons souligner que les deux classes à scripteurs plus faibles, CM2-D et $6^{\mathrm{e}}-\mathrm{C}(1)$, progressent nettement en R3 par rapport au CM2-C témoin. La progression des élèves du CM2-D est même remarquable : $56 \%$ versus $50 \%$. Seule la $6^{\mathrm{e}}-\mathrm{C}$ (2) témoin, ne bénéficiant que d'un rappel et pas de SLO, présente de très faibles performances dans l'emploi du vocabulaire de la ruse.

L'analyse des UL du vocabulaire de la ruse dans les rappels de récit permet de nuancer l'impression de faible efficacité des SLO au regard du réinvestissement dans les écrits d'invention de ruse. Au contraire, leur intérêt semble important, spécialement pour les faibles scripteurs. Nous allons montrer maintenant, en observant plus finement les UL employées par les élèves, que les SLO ont un impact sur le réinvestissement des UL, non pas globalement, mais sur certaines classes grammaticales ou sur la variété des reformulations. 


\subsection{L'analyse qualitative des performances lexicales dans les productions écrites}

Voici les UL dénotant la ruse entendues par les élèves au fil des contes lus. Elles sont constituées de verbes simples, de noms, d'adjectifs ou groupes adjectivaux que nous allons présenter dans leur environnement lexical.

Dans le premier conte, les élèves entendent huit UL :

- les verbes duper et berner: «Lorsque le lion sut comment le lièvre avait dupé la gazelle et l'antilope, [...] », « Le lièvre ne me dupera pas, moi », « Tu te laisseras berner aussi ! ;

- les adjectifs astucieux, naïve, crédule, intelligent et rusée : "Le lièvre astucieux » (titre), "Ainsi la naïve gazelle laissa le lièvre attacher ses pattes de devant », « Et l'antilope crédule laissa le lièvre attacher ses pattes de devant ", "Je suis plus intelligent que tous les autres réunis ", « La grenouille rusée lui attrapa une patte arrière ».

Dans le deuxième conte, les élèves entendent six UL :

- le verbe berner : « dit le chat bien décidé à berner l'ogre» ;

- les adjectifs peu instruit et étourdi : «un jeune lapin, peu instruit encore des ruses de ce monde ", " un jeune étourdi de lapin »;

- les noms ruses, malin et plan: "peu instruit encore des ruses de ce monde », "Le malin, en vérité, les avait cachés sous une grosse pierre», "le chat, ravi de voir que son plan commençait à réussir ».

Dans le troisième conte, les élèves entendent quatre UL :

- le verbe tromper: «Furieux d'avoir été trompé, il courut chez la gazelle », « La gazelle m'a encore trompé ";

- l'adjectif naïve : «L'antilope, qui était naïve, accepta de délier les liens et de prendre sa place dans le filet »;

- le nom ruses : « Les trois ruses de la gazelle » (titre).

7 Les élèves ont donc pu mémoriser des UL et les réemployer à l'identique (ou presque) ou choisir de reformuler, par des unités considérées par eux comme synonymes. Nous proposons ci-dessous l'inventaire des $\mathrm{UL}^{5}$ employées dans les écrits des élèves (rappels et écrits d'invention).

\subsubsection{Les UL qualifiant le trompeur}

Le tableau 4 présente la variété des qualificatifs employés pour le trompeur et les pourcentages arrondis du taux d'emplois par rapport au nombre d'écrits. Les italiques mettent en relief les UL entendues dans les textes sources, les autres sont des UL reformulées par les élèves.

Tableau 4. - Les qualificatifs pour le trompeur employés dans les productions écrites.

\begin{tabular}{|c|c|c|c|c|c|c|c|c|}
\hline \multirow{2}{*}{$\begin{array}{l}\text { Qualificatifs } \\
\text { pour le trompeur }\end{array}$} & \multicolumn{2}{|l|}{ R1 } & \multicolumn{3}{|c|}{ R2 et 3} & \multicolumn{3}{|c|}{ Ruse inventée } \\
\hline & $\begin{array}{l}4 \\
\text { SLO }\end{array}$ & $\begin{array}{l}\text { sans } \\
\text { SLO }\end{array}$ & $\begin{array}{l}4 \\
\text { SLO }\end{array}$ & $\begin{array}{l}\text { sans } \\
\text { SLO }\end{array}$ & $\begin{array}{l}\operatorname{slm} t \\
\text { R3 }\end{array}$ & $\begin{array}{l}4 \\
\text { SLO }\end{array}$ & $\begin{array}{l}\text { sans } \\
\text { SLO }\end{array}$ & $\begin{array}{l}\operatorname{slm} t \\
\text { R3 }\end{array}$ \\
\hline astucieux (conte 1) & 1 & 5 & 0 & 0 & 0 & 0 & 0 & - \\
\hline
\end{tabular}




\begin{tabular}{|l|l|l|l|l|l|l|l|l|}
\hline ce coquin de chat & 0 & 0 & 1 & 0 & 0 & 0 & 0 & 0 \\
\hline plus fort & 1 & 1 & 0 & 0 & 0 & 0 & 0 & 0 \\
\hline futée & 0 & 0 & 1 & 0 & 0 & 0 & 0 & 0 \\
\hline intelligent (conte 1) & 9 & 1 & 2 & 0 & 0 & 0 & 0 & - \\
\hline malicieux & 0 & 1 & 2 & 1 & 0 & 0 & 0 & 0 \\
\hline malin (le malin conte 2) & 8 & 7 & 9 & 4 & 0 & 5 & 0 & 0 \\
\hline pas naïve & 0 & 1 & 0 & 1 & 0 & 0 & 0 & 0 \\
\hline rusée (conte 1) & 7 & 5 & 12 & 1 & 0 & 9 & 0 & - \\
\hline avait plus d'un tour dans son sac & 0 & 0 & 1 & 0 & 0 & 0 & 0 & 0 \\
\hline Nombre de productions écrites & 89 & 23 & 163 & 46 & 16 & 85 & 26 & 16 \\
\hline Nombre d'UL adjectivales \\
Pourcentage d'emplois (arrondi)
\end{tabular}

Pour les UL qualifiant le trompeur, le CM2-C témoin est plus performant que les autres classes dès le départ ( $91 \%$ versus $29 \%$ ). Pourtant, nous pouvons constater que cette supériorité s'estompe en $\mathrm{R} 2$ et disparait dans l'écrit d'invention.

Les qualificatifs pour le trompeur ont été systématiquement travaillés dans les douze SLO, pour rechercher des synonymes déjà connus ou entendus dans les contes. L'inventaire des UL entendues «astucieux, rusée, intelligent, malin » s'est enrichi de «futé, pas bête, sournois, malicieux, roublard ». Les explicitations lexicales ont permis de raffiner le sens de certains adjectifs ou de corriger des acceptions erronées, en particulier « sournois » défini par une élève comme « à moitié sourd ».

\subsubsection{Les UL qualifiant le trompé}

41 Pour les UL qualifiant le trompé, la différence de performances lexicales est moins sensible. Voici l'inventaire des unités lexicales dans les trois rappels écrits; le premier nombre correspond aux emplois des classes à SLO, le deuxième au CM2-C témoin (sans SLO) :

- bête : $1 / 0$

- un petit con de lapin : 1 / 0

- crédule (conte 1) : 2 / 1

- étourdi (conte 2) : 2 / 1

- pas très futé : 1 / 0

- ignorante $: 1$ / 0

- peu instruit des ruses de la vie: 0 / 1

- qui ne connaissait pas les ruses de ce monde dangereux : 1 / 0

- *non expériencé des ruses de la vie : 0 / 1

- sans connaitre les dangers de la nature : 0 / 1 
- non instruit : 0 / 1

- peu malin : 0 / 1

- naï/ve (contes 1,3$): 29$ / 6

- stupide $: 0 / 1$

Nous signalons que la $6^{\mathrm{e}}-\mathrm{C}(2)$ témoin, qui n'a entendu et rappelé que le conte 3 sans SLO, n'a employé aucune qualification des personnages. Nous supposons que la faible imprégnation lexicale en réception est l'un des facteurs explicatifs à cette absence. Dans les écrits d'invention, deux UL ont été employées pour qualifier le trompé :

- naïf/ve (contes 1,3) : 6 / 0

- peu rusé : 0 / 1

43 Les qualificatifs pour le trompé n'ont été travaillés que dans cinq SLO seulement. Quand bien même le taux de réemploi est faible, il faut bien reconnaitre que l'adjectif naïf/ve a connu un relatif succès, uniquement dans les classes à SLO.

\subsubsection{Les UL verbales dénotant la ruse}

L'inventaire des expressions verbales dénotant la ruse ${ }^{7}$ nous montre là encore que le CM2-C témoin est presque aussi performant que les classes à SLO et qu'en revanche, la $6^{e}-C$ (2) témoin a un très faible taux de réemplois. Verbes simples, de niveau de langue courant (piéger, tromper) ou familier (arnaquer, embobiner), côtoient des collocations à base de verbes supports (faire une blague, dire des mensonges) ou collocations opaques (tomber dans le panneau, rouler dans la farine), des erreurs de construction verbale (*ruser quelqu'un, *se faire tromper). Malgré les bons scores de réinvestissement du CM2-C témoin dans l'écrit d'invention, nous constatons que les quatre classes à SLO emploient des UL verbales plus variées (seize items contre douze pour la classe témoin).

\section{Les retombées du dispositif didactique sur les élèves et les enseignants}

\subsection{Des bénéfices pour des élèves en production écrite ou en séance orale}

Dans toutes les classes, des élèves faibles scripteurs se sont impliqués lors des séances lexicales orales, pour donner leur avis sur tel ou tel emploi d'un verbe ou d'un adjectif, pour proposer un synonyme, etc. Les enseignantes participant à l'expérimentation se sont réjouies de la participation d'élèves en difficulté lors de ces séances.

Cécile, en charge de la $6^{\mathrm{e}}-\mathrm{C}$, évoque par exemple la "révélation» de Gabin, " généralement effacé à l'oral et improductif à l'écrit ». Les séances filmées le montrent dynamique et très coopératif. Abdou, très faible lecteur au comportement difficile, qui a refusé d'écrire les rappels, est intervenu timidement dès la première séance orale et a été attentif aux échanges de ses camarades.

Anne-Laure, en charge de la $5^{\mathrm{e}}$, nous a signalé l'exemple de Warren qui s'est montré particulièrement actif par rapport à son attitude habituelle. Il est décrit comme un élève faible, perturbateur, mais peu à peu plus enclin au travail vers le milieu du deuxième trimestre. Lors de la troisième séance orale, il intervient spontanément à vingt reprises pour répondre à une question ouverte à tous en proposant un mot, une 
phrase courte, plusieurs phrases ou segments propositionnels. Il se lance, pendant près de deux minutes, dans un rappel oral du conte, encouragé par l'enseignante. Malgré ses difficultés, Warren mène jusqu'au bout son récit.

Parallèlement aux séances orales qui constituent le cœur du dispositif, les enseignantes ont découvert l'intérêt du rappel de récit écrit, phase qui a permis de collecter des données pour analyser des emplois lexicaux et développer des interactions orales. Selon la formule de Clarice, enseignante du CM2-témoin, «le rappel de récit entendu est un véritable lanceur d'écriture notamment pour les faibles scripteurs». Le cas le plus remarquable est celui de Shaïna (CM2-C témoin) décrite par son enseignante comme une élève en grande difficulté, incapable de produire un texte. La comparaison des quatre productions écrites montre une réelle progression : R1 (51 mots), R2 (76 mots), R3 (192 mots), écrit d'invention ( 150 mots). Les rappels sont certes incomplets et maladroits, mais Shaïna a été fière d'avoir participé et d'avoir réussi à écrire comme les autres élèves de sa classe. Son enseignante nous a rapporté la question de Shaïna quelque temps après : « Maitresse, c'est quand qu'on écrit ? »

\subsection{Des bénéfices sur les pratiques professionnelles}

Les enseignantes ont découvert une nouvelle façon d'entrer collectivement dans la compréhension d'un récit en abordant le vocabulaire. Le bilan de leurs ressentis et des bénéfices constatés est satisfaisant, voire très satisfaisant.

Avant même le commencement de l'expérimentation, les enseignantes ont manifesté leur inquiétude face à une séance exclusivement orale, sans trace écrite pour les élèves, durant environ 45 minutes. Pour rassurer les enseignantes inquiètes de ne pas avoir de support matériel pour canaliser l'attention des élèves, nous les avons autorisées à noter au tableau la trace des "trouvailles", sous la forme de leur choix. Les élèves ne devaient rien noter pour préserver la dominante orale du travail lexical. Chacune a choisi de noter les mots des élèves au fur et à mesure sous forme de listes par classes grammaticales, ou sous la forme d'une constellation partant d'un cœur thématique « la ruse » et des traits rattachant les mots à l'hyperthème.

51 Cécile $\left(6^{e}-\mathrm{C}\right)$, par exemple, a pris l'initiative de projeter les phrases comportant des UL collectées. Elle reconnait que l'expérience a été «inconfortable au départ par son caractère inédit, mais fructueuse avec le recul, surtout lorsqu'on se rend compte de l'incroyable quantité de notions abordées en une seule séance et de la finesse de la réflexion des élèves ».

Danielle (CM2-D), témoigne, avec beaucoup de lucidité, de son trouble pour la préparation des séances orales. Elle écrit dans le questionnaire-enquête: «Pour la préparation de la séance orale, n'étant pas habituée à ce genre d'activité, j'étais un peu perdue. J'ai trouvé difficile d'élaborer les questions.»Certes, le travail préparatoire nécessite beaucoup de temps pour sélectionner des items pertinents, mais la production écrite est une médiation efficace pour " parler sur ${ }^{8}$ » des choix lexicaux réels, qui feront sens pour les élèves puisque leurs auteurs seront identifiés. Cette reconnaissance de « l'auteur » est à la fois une valorisation pour les UL réussies et une aide pour les UL maladroites. Pour que ces activités langagières réduisent les inégalités, les enseignants doivent accompagner les élèves et organiser des situations de travail à partir des pratiques des élèves et de leurs différences. 


\section{Conclusion}

53 Notre dispositif didactique articule l'enseignement-apprentissage d'unités lexicales dénotant la ruse à la lecture, à des activités réflexives orales et à la production écrite. Nous avons, en quelque sorte, ouvert une partie du «chantier» de l'« apprentissage intégré » qu'évoque Grossmann (2018, p. 10). Le va-et-vient entre l'oral, la lecture et l'écrit est interrogé au prisme des choix lexicaux dénotant la ruse et les qualités intrinsèques des personnages trompeurs et trompés. Les analyses montrent des disparités de performances lexicales entre les six classes, corrélées vraisemblablement au contenu variable des séances orales ou aux aléas rencontrés lors de la passation. Cependant, ces constats n'invalident pas le projet dans son ensemble. Si l'impact global de la répétition d'activités orales sur le réinvestissement du vocabulaire en écriture d'invention de ruse n'est pas probant, les bénéfices apparaissent dans le troisième rappel écrit. Nous avons constaté la nette progression des performances lexicales des deux classes plus faibles. Plus largement, les bénéfices du dispositif touchent la modification du rapport de certains élèves aux activités d'apprentissage et au choix des mots. Le témoignage des enseignantes révèle la motivation et l'implication renforcée des faibles scripteurs ou faibles parleurs. Nous envisageons d'analyser plus finement dans d'autres travaux la qualité des interactions verbales enseignant-élèves(s) et entre élèves pour suggérer des pistes d'amélioration. Tous ces éléments nous incitent à proposer aux enseignants d'intégrer des séances lexicales orales dans leurs activités pour développer des compétences lexicales, mais aussi affiner la compréhension en lecture et sensibiliser les élèves au réinvestissement lexical en production écrite.

\section{BIBLIOGRAPHIE}

BAUTIER, Élisabeth. (2016). Et si l'oral pouvait permettre de réduire les inégalités ? Les dossiers des sciences de l'éducation, 36, 95-108.

BRIGAUDIOT, Mireille. (2000). Apprentissages progressifs de l'écrit à l'école maternelle. Paris : Hachette.

DENHIÈRE, Guy \& BAUDET, Serge. (1992). Lecture, compréhension de texte et science cognitive. Paris :

PUF.

GaRcia-Debanc, Claudine \& Plane, Sylvie. (2004). Comment enseigner l'oral à l'école primaire? Paris : INRP-Hatier.

GIASSON, Jocelyne. (1996). La compréhension en lecture. Bruxelles : De Boeck.

GoIGOUX, Roland \& CÈBE, Sylvie. (2018). Lectorino \& Lectorinette. Apprendre à comprendre les textes narratifs. Paris : Retz.

GRANDATY, Michel \& TURCO, Gilbert. (2001). L'oral dans la classe. Discours, métadiscours, interactions verbales et construction de savoirs à l'école primaire. Paris : INRP. 
GROSSMANN, Francis. (2018). Comment intégrer l'enseignement du lexique dans la production écrite?

Disponible en ligne sur <www.cnesco.fr/wp-content/uploads/2018/04/

CCEcrits_note_Grossmann.pdf>.

Grossmann, Francis \& Boch, Françoise. (2003). Production de textes et apprentissage lexical :

l'exemple du lexique de l'émotion et des sentiments. Repères, 28, 117-135.

GROSSMANN, Francis \& PlANE, Sylvie. (2008). Les apprentissages lexicaux. Lexique et production verbale. Villeneuve-d'Ascq : Presses universitaires du Septentrion.

HAYES, John-Richard \& FLOWER, Linda. (1980). Identifying the Organisation of Writing Processes.

Dans L. W. Gregg \& E. R. Stienberg (dir.), The Dynamics of Composing. Cognitive Processes in Writing (p. 3-30). Hillsdale, NJ : LEA.

JoHnSON-LAIRD, Philip. (1983). Mental Models: Toward a Cognitive Science of Language. Cambridge, MA : Cambridge University Press.

LE NY, Jean-François. (1989). Science cognitive et compréhension du langage. Paris : PUF.

VAN DIJK, Teun \& KINTSCH, Walter. (1983). Strategies of Discourse Comprehension. New York :

Academic Press.

VÉNÉRIN-GUÉNEZ, Christine. (2020). Développer les compétences lexicales en français à La Réunion. De l'oral à l'écrit et inversement. Dans S. Genevois \& N. Wallian (dir.), Enseigner-apprendre en tous terrains : de la didactique contextuelle à la contextualisation du didactique (p. 127-145). Paris : Éditions des archives contemporaines. Disponible en ligne sur <https://eac.ac/books/9782813003492>.

VÉNÉRIN-GUÉNEZ, Christine. (2018a). À la recherche de stratégies scripturales dans des rappels de récit écrits par des élèves de cours moyen. Repères, 57, 83-97. Disponible en ligne sur <https:// journals.openedition.org/reperes/1488>.

VÉNÉRIN-GUÉNEZ, Christine. (2018b). Dynamiques d'entretiens métalexicaux avec des élèves de primaire (9-11 ans). La lettre de l'AIRDF, 64, 26-30.

VÉNÉRIN-GuÉNEZ, Christine. (2017). Les choix lexicaux des élèves révélateurs d'un déjà-là. Pratiques, 173-174. Disponible en ligne sur <https://journals.openedition.org/pratiques/3383>.

VÉNÉRIN-GUÉNEZ, Christine. (2012). Mémoire narrative et performances lexicales. Pratiques, 155-156, 147-168. Disponible en ligne sur <http://journals.openedition.org/pratiques/3494>.

VÉNÉRIN-GuÉNEZ, Christine \& GAILLAT, Thierry. (2018). Explicitation lexicale orale en cours moyen : influences du contexte sociolinguistique réunionnais. Dans N. Wallian (dir.), Intervention éducative et médiation(s) (p. 203-226). Berne : Peter Lang.

\section{NOTES}

1. Le conte «Les trois ruses de la gazelle " a servi également de texte source pour le dispositif expérimental de doubles rappels de récit mené de 2011 à 2015, au laboratoire STIH (ParisSorbonne).

2. L'ordre ne suit pas strictement la progression de longueur des contes. En effet, lors de la réunion préparatoire, une enseignante, consciente des difficultés scripturales de ses élèves, a souhaité que l'écriture d'invention d'une ruse soit davantage guidée. Nous avons donc modifié l'ordre des contes, et le sujet d'écriture d'invention a porté sur une quatrième ruse de la gazelle, dernier conte entendu et rappelé. 
3. Le temps d'écriture peut paraitre long, mais un dispositif expérimental de doubles rappels du même récit mené entre 2011 et 2015 dans des classes parisiennes a montré que les élèves disaient très souvent avoir manqué de temps pour raconter, soit en l'exprimant oralement à leur professeur, soit en l'écrivant à la fin de leur texte : «je n'ai pas eu le temps de terminer », « je n'ai pas terminé mais je sais la fin ».

4. "Le chat botté " a connu moins de succès, jugé «long et compliqué » par les élèves (voir supra). Les $\mathrm{R} 2$ sont plus courts, il y a moins de récits complets et davantage de rappels confus ou erronés par rapport aux deux autres rappels.

5. Dans le cadre restreint de cette contribution, nous n'aborderons pas les UL nominales utilisées en plus faible proportion hors des expressions verbales (astuces, blague, malin, mensonges, piège, plan, ruses).

6. Nous intégrons la collocation verbale qui s'apparente sémantiquement à une qualification implicite.

7. L'inventaire des expressions verbales dénotant la ruse: arnaquer, avoir quelqu'un, se faire / se laisser avoir, berner, se faire berner / se laisser berner / être berné, faire une blague, faire le coup de / réussir son coup, duper, se faire duper / être dupé, embrouiller, entourlouper, faire une farce, mentir / dire des mensonges / dire des bobards, piéger, se faire piéger / être piégé, tomber dans le panneau, tomber dans le piège, faire / mener une tactique, verbe + un piège, verbe + un plan, verbe + une ruse, le plan commençait à réussir / le plan / le piège / le stratagème fonctionne / marche / réussit, rouler (quelqu'un), rouler dans la farine, *ruser (quelqu'un) / ruser (intransitif), avoir recours à un stratagème, avoir une ruse / un plan en tête / derrière la tête, jouer un (nouveau) tour, préparer un mauvais tour, tromper, être trompé / *se faire tromper.

8. Nous empruntons cette notion de « travail sur » à Bautier (2016).

\section{RÉSUMÉS}

Nous rendons compte d'un dispositif didactique mis en œuvre dans six classes, sur trois niveaux $\left(\mathrm{CM} 2,6^{\mathrm{e}}, 5^{\mathrm{e}}\right)$, entre février et mai 2018. Notre recherche vise à mesurer l'influence de plusieurs séances lexicales orales sur le réinvestissement en production écrite. Les unités lexicales sur le thème de la ruse sont sélectionnées dans des rappels écrits de contes entendus. Le dispositif favorise l'activité réflexive sur les choix lexicaux, en réception et en production, donc le développement de la compétence lexicale. Le bilan des bénéfices sur les performances lexicales des élèves et leur motivation est très encourageant.

We report on a didactic experiment carried on six classes, on three levels, between February and May 2018. Our research aims to measure the impact of several oral-based lexical teaching sessions on vocabulary reuse in written work. Lexical units on the theme of trickery are selected from written recall of tales told orally. The experiment encourages thought as to lexical choices, in reception and in production, and therefore the development of lexical skills. The results with regard to pupils' lexical performance and motivation are very encouraging. 
INDEX

Mots-clés : compétences lexicales, vocabulaire de la ruse, interactions orales, production écrite Keywords : lexical skills, vocabulary of trickery, oral interactions, written work

\section{AUTEUR}

\section{CHRISTINE VÉNÉRIN-GUÉNEZ}

Laboratoire Icare - EA 7389, Université de La Réunion 